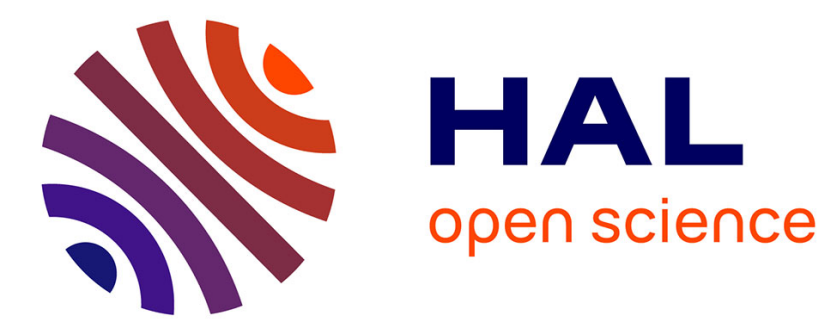

\title{
Optimism, pessimism and financial bubbles
}

Bertrand Wigniolle

\section{To cite this version:}

Bertrand Wigniolle. Optimism, pessimism and financial bubbles. 2012. halshs-00673892

\section{HAL Id: halshs-00673892 \\ https://shs.hal.science/halshs-00673892}

Submitted on 24 Feb 2012

HAL is a multi-disciplinary open access archive for the deposit and dissemination of scientific research documents, whether they are published or not. The documents may come from teaching and research institutions in France or abroad, or from public or private research centers.
L'archive ouverte pluridisciplinaire HAL, est destinée au dépôt et à la diffusion de documents scientifiques de niveau recherche, publiés ou non, émanant des établissements d'enseignement et de recherche français ou étrangers, des laboratoires publics ou privés. 


\section{Documents de Travail du Centre d'Economie de la Sorbonne}

U

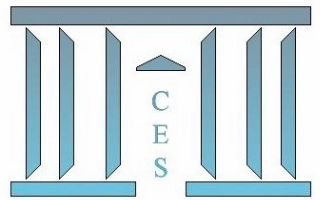

Optimism, pessimism and financial bubbles

Bertrand WigNiOLLE

2012.05

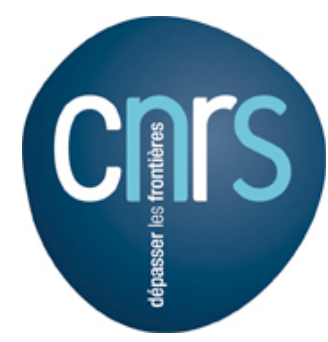

Maison des Sciences Économiques, 106-112 boulevard de L'Hôpital, 75647 Paris Cedex 13 http://centredeconomiesorbonne.univ-paris1.fr/bandeau-haut/documents-de-travail/ ISSN : 1955-611X 


\title{
Optimism, pessimism and financial bubbles*
}

\author{
B. Wigniolle ${ }^{\dagger}$
}

February 2, 2012

*I am grateful to Michèle Cohen, Mégléna Jéléva and Jean-Christophe Vergnaud for stimulating discussions on the RDU model. I thank the participants at the conference "New challenges for macroeconomic regulation: financial crisis, stabilisation policy and sustainable development", Marseille 2011, for their helpful comments, and particularly Lise Clain-Chamosset, Thomas Seegmuller and Robert Becker. This paper was also presented at the 11th SAET Conference. I thank Jean-Marc Bonnisseau for his invitation and the participants at his session for their comments.

${ }^{\dagger}$ Paris School of Economics, University of Paris 1 Pantheon-Sorbonne. Address: C.E.S., 106-112, boulevard de l'hôpital, 75647 Paris Cedex 13, France. Tel: +33 (0)1 44078198. Email : wignioll@univ-paris1.fr. 


\begin{abstract}
:
This paper shows that it is possible to extend the scope of the existence of rational bubbles when uncertainty is introduced associated with rank-dependent expected utility. This RDU assumption can be viewed as a transformation of probabilities depending on the pessimism/optimism of the agent. The results show that pessimism favors the existence of deterministic bubbles, when optimism may promote the existence of stochastic bubbles. Moreover, under pessimism, the RDU assumption may generate multiple bubbly equilibria. The RDU assumption also leads to new conditions ensuring the (absence of) Pareto-optimality of the competitive equilibrium without bubbles. These conditions still govern the existence of bubbles. JEL classification: D81, D9, G1
\end{abstract}

Keywords: rational bubbles, RDU preferences. 


\section{Introduction}

This paper shows that the scope for the existence of rational bubbles can be extended when uncertainty and rank-dependent expected utility are introduced. In the framework of an overlapping generations model à la Diamond (1965), the seminal article by Tirole (1985) proves that bubbles can arise in economies for which the return on capital at steady state is below the growth rate of output. The bubbleless economy must be in a state of overaccumulation that corresponds to dynamic inefficiency. Weil (1987) proposes a model of stochastic bubbles using the same framework as Tirole, and finds existence conditions that are even stronger. Different authors have introduced rational bubbles in richer frameworks with endogenous growth (e. g. Grossman and Yanagawa, 1993 and Olivier, 2000). But the existence of bubbles remains linked to the same condition between the growth rate and the interest rate. As empirical observations suggest that this condition is not fulfilled in general (see Abel et alii, 1989), rational bubbles seem unlikely to arise. They may perhaps not be the pertinent explanation to understand bubble phenomena that actually are observed.

In recent contributions, Caballero and Hammour (2002) and Caballero, Farhi and Hammour (2006) obtain the existence of bubbles under less stringent conditions at the price of a transformation of the notion of bubble. They build an overlapping generations model with an adjustment cost to capital leading to two long-run equilibria. They interpret the equilibrium corresponding to a higher valuation of the capital stock as a bubbly equilibrium.

This paper intends to show that it is possible to extend the scope for the existence of rational bubbles when uncertainty is introduced associated with a rank-dependent expected utility. A simple overlapping generations model is studied in which the production technology depends on a technological shock: capital return is random. In order to have a very tractable model, there are only two possible states of the nature and the capital return may oscillate between a high and a low value. In an economy in which capital is the only asset, financial markets are incomplete as two states of the nature exist. The existence of a bubbly asset can make financial markets complete. Two types of bubbles are considered in this context. The first type, called 
a deterministic bubble, is an asset that has the same price in both states of the nature. The second type, called a stochastic bubble, is an asset whose existence is conditional to the occurrence of a particular state of the nature. As in Weil (1987), agents form their expectations according to a self-fulfilling prophecy which assumes that the bubble will burst if the other state arises.

Moreover, it is assumed that agents are endowed with a rank-dependent expected utility (RDU) function. This model has been introduced by Quiggin (1993) and developed by Chateauneuf (1999). A general presentation can been found in Cohen and Tallon (2000). According to the RDU model, the distribution of probabilities is transformed by a probability weighting function (pwf). The utility is no longer linear with respect to the probabilities of the states of nature. This assumption can be viewed as a transformation of probabilities depending on the pessimism/optimism of the agent. A pessimistic agent will give more weight to the bad state of the nature, whereas an optimistic agent will give more weight to the good state. This assumption has two implications. Firstly, the deformation of probabilities may lead to quantitative changes with respect to the ones obtained with the standard EU (Expected Utility) model. More precisely, our results show that pessimism favors the existence of deterministic bubbles and of small stochastic bubbles, while optimism may promote the existence of big stochastic bubbles. Secondly, qualitative changes may arise related to the property that the weighting of the different states can change when the associated gains are modified: this property can lead to a multiplicity of bubbly equilibria.

Considering pessimistic agents in the case of a deterministic bubble, the transformation of probabilities weakens the existence conditions of a bubble. The interpretation is simple. By assumption, the gross capital return is greater than 1 in the good state of the nature, while it is smaller than 1 in the bad state. Investing in the bubble provides a gross return equal to 1. Agents invest in the bubble in order to be protected against the occurrence of the bad state. In the case of pessimism, they put more weight on this state and invest more in the bubble. Therefore, pessimism may support the bubble.

Considering the case of a stochastic bubble, the transformation of probabilities may weaken the existence conditions of a bubble. Assuming that the 
existence of the bubble is conditional to the state with a low capital return, the bubble bursts if the state with a high capital return emerges. Therefore, two types of bubbly equilibria may exist, associated with a low value or a high value of the bubbly asset. If the value of the bubble is low, the state with a high capital return remains the good state for the consumer. But if the value of the bubble is high, it is possible that the state with a low capital return becomes the good state for the consumer. As the consumer assigns different weights to these two cases, two types of equilibria may exist associated with a low value or a high value of the bubble.

In the case of a high value of the bubble, optimism promotes the existence of this type of equilibrium. As the good state for the consumer corresponds to the existence of the bubble, optimistic agents assign more weight to the bubbly state and invest more in the bubble. In the end, optimism favors stochastic bubbles.

In contrast, the existence of the equilibrium with a low value of the bubble is favored by pessimism. Indeed, when the value of the bubble is low, the bubbly state is the bad state of nature for the consumer. A pessimistic agent assigns more weight to this state, which favors the existence of a bubbly equilibrium.

In the case of pessimism and stochastic bubbles, an equilibrium may exist associated with a value of the bubble such that the two states of nature lead to equal levels of consumption. Moreover, this bubbly steady state may be stable and there is convergence with oscillations. There exists an infinity of initial conditions for the value of the bubble and the bubbly equilibrium is indeterminate. The existence of such an equilibrium is due to the "kink" in the indifference curves which appears for equal levels of consumption, in the two states of nature in the RDU framework.

This result can be related to previous ones obtained in finance literature with rank dependant utility or Choquet utility: Tallon (1997) and Epstein and Wang (1994) also obtain the existence of multiple equilibria. The originality of this work is to obtain the result in a production economy with capital and a bubbly asset. Bosi and Seegmuller (2010) have also developed a framework in which there exists an indeterminate bubbly equilibrium. In their model, indeterminacy is due to frictions introduced via a cash-in-advance 
constraint with financial market imperfections.

This paper successively considers different assumptions: EU preferences, RDU preferences, deterministic bubbles, and stochastic bubbles. For each case, the existence conditions of bubbles are related to the Pareto optimality properties of the economy without bubbles. As expected, bubbles can only appear in an economy for which the competitive equilibrium is not Pareto optimal. For RDU preferences, the condition ensuring Pareto optimality also depends on the transformation of probabilities implied by the pessimism/optimism of the agents. Therefore, it cannot be reduced to a comparison between the interest rate and the growth rate of the economy. It provides an additional degree of freedom that may reconcile the existence of bubbles with parameters that are empirically relevant. The study of Pareto optimality in the paper is based particularly on previous articles that have studied Pareto optimality of allocations in overlapping generations models with stochastic shocks, such as Peled (1984), Peled and Aiyagari (1991), Wang (1993), Gottardi (1996) and Demange and Laroque (1999).

Section 2 presents the basic framework with EU preferences and a competitive equilibrium without bubbles. A condition ensuring the Pareto optimality of this equilibrium is derived. Section 3 studies the existence of deterministic and stochastic bubbles in this framework. Section 4 introduces RDU preferences and derives new existence conditions for bubbles. Section 5 concludes. Appendixes are presented in Section 6

\section{The basic model}

\subsection{The competitive equilibrium without bubbles}

The basic setup is an overlapping generations model with capital accumulation à la Allais (1947)-Samuelson (1958)-Diamond (1965). Agents live during two periods. They supply one unit of labor in the first period (when young) and they are retired and consume the proceeds of their savings in the second period (when old). The number of agents in each generation is normalized to 1 .

There is a single good in the economy, produced in period $t$ with capital 
$K_{t-1}$ (the capital stock results from $t-1$ savings) and labor $L_{t}$. In each period $t$ exists one competitive firm using a linear production technology

$$
Y_{t}=R\left(\sigma_{t}\right) K_{t-1}+w L_{t}
$$

Capital depreciation is completed in one period. Labor productivity is constant and equal to $w$. Capital productivity $R\left(\sigma_{t}\right)$ follows a random process that depends on the state of the nature $\sigma_{t}$. At each period $t, \sigma_{t} \in\{1,2\}$. State 1 occurs with probability $\pi$ and state 2 with probability $1-\pi$. For $\sigma_{t}=i, i=1,2, R(i)$ will be denoted $R_{i}$ and it is assumed that

$$
R_{1}>1>R_{2}
$$

In each period $t, \sigma_{t}$ is known by agents before they make their choices. Under perfect competition, it is straightforward that $w$ will be the equilibrium value of the wage and $R\left(\sigma_{t}\right)$ the capital gross rate of return.

An agent born in $t$ knows the state of the economy for $t$, but not for $t+1$. $c_{t}$ is the first period consumption, $s_{t}$ is the amount of savings, and $d_{t+1}$ is the second period consumption that is a random variable in $t$. The budget constraints of the agent are:

$$
\begin{aligned}
c_{t}+s_{t} & =w \\
d_{t+1} & =R\left(\sigma_{t+1}\right) s_{t}
\end{aligned}
$$

The agent is endowed with an intertemporal utility function. The utility function of a generation $t$ agent is:

$$
\ln c_{t}+\beta E_{t} \ln \left(d_{t+1}\right)
$$

$E_{t}$ is the expectation taken in period $t$. Maximizing (3) under budget constraints (1) and (2) gives the results:

$$
\begin{aligned}
c_{t} & =\frac{w}{1+\beta} \\
s_{t} & =\frac{\beta w}{1+\beta} \\
d_{t+1} & =\frac{R\left(\sigma_{t+1}\right) \beta w}{1+\beta}
\end{aligned}
$$


Results do not depend on $\pi$ as the VNM function is logarithmic.

The capital stock used in period $t+1$ results from savings in $t$ :

$$
K_{t}=\frac{\beta w}{1+\beta}
$$

Therefore, after one period, the capital stock reaches a constant value.

In $t=0$, the initial value of the capital stock $K_{-1}$ is given, as the consumption level of the first old agent: $d_{0}=R\left(\sigma_{0}\right) K_{-1}$.

\subsection{Pareto optimality of the competitive path}

A standard result in deterministic models is that the existence of financial bubbles is possible only in economies that are inefficient. If uncertainty is removed from the model $(R(1)=R(2)=R)$, dynamic efficiency is obtained for $R>1$.

In an economy with uncertainty, the appropriate concept of Pareto optimality for allocation is interim optimality (see Demange and Laroque (1999)). A feasible allocation is interim optimal if no other feasible allocation exists that, almost surely, yields a higher expected utility for all periods $t$ with a strict improvement on a set of states of positive measure.

Interim Pareto optimality can be translated in this framework as follows. Firstly, the notion of feasible path is introduced. In period $t$, the resource constraint of the economy can be expressed as:

$$
c_{t}+d_{t}+K_{t}=R\left(\sigma_{t}\right) K_{t-1}+w
$$

A feasible path is an allocation $\left(c_{t}, d_{t}, K_{t}\right)_{t \geq 0}$, starting from a given value for $K_{-1}$, that satisfies the resource constraint (4) for all $t$.

\section{Proposition 1 If}

$$
\frac{\pi}{R_{1}}+\frac{1-\pi}{R_{2}}<1
$$

the competitive equilibrium is interim Pareto optimal.

Proof. See Appendix 1. 
The proposition shows that interim Pareto optimality is preserved if the low value of $R_{2}$ in the bad state is compensated by a high enough value of $R_{1}$ in the good state. It is interesting to note that if (5) holds, then $\pi R_{1}+(1-\pi) R_{2}>1$. Therefore, Pareto optimality needs a stronger condition than an average interest rate higher than the growth rate of capital.

\section{The equilibrium with financial bubbles}

This section assumes the existence of a bubble asset. This asset is a pure bubble, with a fundamental value equal to 0 . Two types of bubbles are studied: one is called a bubble "à la Tirole" which is "deterministic", while the second is a bubble "à la Weil" which is "stochastic". The deterministic bubble is a deterministic asset that has the same price in the two states of the nature. The stochastic bubble only exists in state 2 (the bad state of the nature). Its existence is therefore conditional to the continuation of this state and at each period, the bubble has a probability $\pi$ of exploding in the next period.

\subsection{Existence of an equilibrium with a deterministic bubble}

It is assumed that a bubble asset is available in the economy in a fixed quantity normalized to 1 . Its price is $p_{t}$ in period $t$. The budget constraints of a generation $t$ agent become:

$$
\begin{aligned}
c_{t}+s_{t}+p_{t} x_{t} & =w \\
d_{t+1} & =R\left(\sigma_{t+1}\right) s_{t}+p_{t+1} x_{t}
\end{aligned}
$$

$x_{t}$ is the demand for the bubble asset. 
Maximizing (3) under budget constraints (6) and (7) gives the results:

$$
\begin{aligned}
c_{t} & =\frac{w}{1+\beta} \\
s_{t} & =\frac{\beta w}{1+\beta}\left(\frac{\pi}{1-\frac{p_{t} R_{2}}{p_{t+1}}}-\frac{1-\pi}{\frac{p_{t} R_{1}}{p_{t+1}}-1}\right) \\
p_{t+1} x_{t} & =\frac{\beta w}{1+\beta}\left[\frac{(1-\pi) R_{1}}{\frac{p_{t} R_{1}}{p_{t+1}}-1}-\frac{\pi R_{2}}{1-\frac{p_{t} R_{2}}{p_{t+1}}}\right]
\end{aligned}
$$

Equilibrium conditions on the bubble and capital markets imply:

$$
\begin{aligned}
x_{t} & =1 \\
K_{t} & =s_{t}
\end{aligned}
$$

Condition (11) with (10) gives the dynamics of the price of the bubble:

$$
p_{t+1}=\frac{\beta w}{1+\beta}\left[\frac{(1-\pi) R_{1}}{\frac{p_{t} R_{1}}{p_{t+1}}-1}-\frac{\pi R_{2}}{1-\frac{p_{t} R_{2}}{p_{t+1}}}\right]
$$

This dynamics has two steady states, 0 which is stable and a positive stationary state $p$ that is unstable:

$$
p=\frac{\beta w}{1+\beta}\left[\frac{(1-\pi) R_{1}}{R_{1}-1}-\frac{\pi R_{2}}{1-R_{2}}\right]
$$

At this stationary state, the value of savings is:

$$
s=\frac{\beta w}{1+\beta}\left(\frac{\pi}{1-R_{2}}-\frac{1-\pi}{R_{1}-1}\right)
$$

Finally, this equilibrium exists only if $p$ and $s$ are positive, which gives:

$$
\begin{aligned}
\frac{\pi}{R_{1}}+\frac{(1-\pi)}{R_{2}} & >1 \\
\pi R_{1}+(1-\pi) R_{2} & >1
\end{aligned}
$$

When the second condition (14) does not hold, it is straightforward enough to show that a bubbly equilibrium exists without investment in capital. This equilibrium is associated with a constant value of the bubble:

$$
p=\frac{\beta w}{1+\beta}
$$


The existence of this equilibrium is related to the assumption of a linear technology, that allows production to occur without using capital.

The results can be summarized by a proposition:

Proposition 2 If condition (13) holds, there exists an equilibrium of the economy associated with a deterministic bubble.

- If (14) is satisfied, agents hold both capital and the bubble asset at equilibrium.

- If (14) is not satisfied, a bubbly equilibrium exists with no investment in capital.

Condition (13) is the converse of the condition that ensures interim Pareto optimality of the competitive equilibrium. As expected, a bubbly equilibrium can only exist in an economy that is not interim Pareto optimal.

When (14) is not fulfilled, two different equilibria may exist: a first one with capital and no bubble, a second one without capital and with the bubbly asset. This second bubbly equilibrium Pareto-dominates the first one (in the sense of interim Pareto optimality). Indeed, with the bubbly equilibrium there is no risk on the gross return of the asset that is equal to 1 , and 1 is higher than the expected return with the equilibrium with capital ( $1>\pi R_{1}+$ $\left.(1-\pi) R_{2}\right)$. Figure 1 gives an illustration of the different cases depending on the values of $R_{1}$ and $R_{2}$. The curves are drawn for the value $\pi=1 / 2$.

Remark 1 This paper focuses only on two types of bubbles: a deterministic one that has the same value in all states of the nature; a stochastic one that cancels out if state 1 occurs. More generally, the case of a bubbly asset that takes two positive different values depending on the state of the nature could be studied. It is easy to check that this type of solution does not exist in our framework.

\subsection{Existence of an equilibrium with a stochastic bub- ble}

A bubble asset à la Weil (1987) is available in the economy in a fixed quantity normalized to 1 . Its price is $p_{t}$ in period $t$, conditional to the realization of 
state 2. Assuming that the economy is in period $t$ in state 2, agents expect a price $p_{t+1}$ in period $t+1$ conditional to the realization of state 2 , and a price 0 if state 1 occurs. In the case of the bubble exploding, the dynamics of the economy after the explosion becomes the same as in the economy without the bubble.

Assuming that state 2 occurs in period $t$, the budget constraints of a generation $t$ agent are:

$$
\begin{aligned}
c_{t}+s_{t}+p_{t} x_{t} & =w \\
d_{t+1}^{1} & =R_{1} s_{t} \\
d_{t+1}^{2} & =R_{2} s_{t}+p_{t+1} x_{t}
\end{aligned}
$$

where $d_{t+1}^{1}$ is the consumption level in state 1 and $d_{t+1}^{2}$ in state 2 .

Maximizing (3) under budget constraints (15), (16) and (17) gives the results:

$$
\begin{aligned}
c_{t} & =\frac{w}{1+\beta} \\
s_{t} & =\frac{\beta w}{1+\beta} \frac{\pi}{1-\frac{p_{t} R_{2}}{p_{t+1}}} \\
x_{t} & =\frac{\beta w}{1+\beta}\left[\frac{(1-\pi)}{p_{t}}-\frac{\pi R_{2}}{p_{t+1}-p_{t} R_{2}}\right]
\end{aligned}
$$

Equilibrium conditions on the bubble market $\left(x_{t}=1\right)$ and on the capital market $K_{t}=s_{t}$ imply:

$$
\begin{aligned}
1 & =\frac{\beta w}{1+\beta}\left[\frac{(1-\pi)}{p_{t}}-\frac{\pi R_{2}}{p_{t+1}-p_{t} R_{2}}\right] \\
K_{t} & =\frac{\beta w}{1+\beta} \frac{\pi}{1-\frac{p_{t} R_{2}}{p_{t+1}}}
\end{aligned}
$$

With the change of variable $\delta_{t}=p_{t}(1+\beta) /(\beta w)$, equation (18) gives:

$$
\delta_{t+1}=R_{2} \delta_{t} \frac{1-\delta_{t}}{1-\pi-\delta_{t}}
$$

This equation has 2 stationary states: 0 which is stable, and

$$
\delta=\frac{1-\pi-R_{2}}{1-R_{2}}
$$


which is unstable. This last steady state exists only if $\delta>0$,

$$
R_{2}<1-\pi
$$

Proposition 3 If condition (20) holds, there exists an equilibrium of the economy associated with a stochastic bubble conditional to the continuation of state 2.

This condition is stronger than (13): the deterministic bubble is more likely to exist than the stochastic bubble. Indeed, the stochastic bubble has a positive return only if state 2 arises. Moreover, the stochastic bubble cannot exist in an efficient economy: if (20) holds, (5) does not hold. A graphical illustration of these results is shown in Figure 1, with a value $\pi=1 / 2$.

\section{Bubbles with a rank-dependent utility}

\subsection{Preferences}

The agent is now endowed with an intertemporal rank-dependent expected utility (RDU) function. Following Quiggin (1993), the distribution of probabilities is transformed by a probability weighting function (pwf) $\phi . \phi$ is a continuous increasing function from $[0,1]$ to $[0,1]$ such that $\phi(0)=0$ and $\phi(1)=1$.

With this assumption, the preceding utility function (3) must be transformed according to the following rules.

Denoting $c, d^{1}$ and $d^{2}$, the consumption levels when young, when old in the state 1 and old in the state 2 , the utility function is:

- if $d^{1}>d^{2}$,

$$
\ln c+\beta \phi(\pi) \ln \left(d^{1}\right)+\beta[1-\phi(\pi)] \ln \left(d^{2}\right)
$$

- if $d^{1}<d^{2}$,

$$
\ln c+\beta[1-\phi(1-\pi)] \ln \left(d^{1}\right)+\beta \phi(1-\pi) \ln \left(d^{2}\right)
$$


In abbreviated form, the utility function will be denoted by:

$$
\ln c+\beta E_{\phi} \ln (d)
$$

where $E_{\phi}$ is the expected value calculated with the transformed probabilities according to $(21)$ and $(22)$, and $d$ is the random variable $\left(d^{1}, d^{2} ; \pi, 1-\pi\right)$.

The property $\phi(\pi)<\pi$ can be interpreted as pessimism and $\phi(\pi)>\pi$ as optimism. An optimistic agent puts more weight on the best state of the nature, whereas a pessimistic agent puts more weight on the worst state.

The following notations will be used from now: $\pi_{1}=\phi(\pi)$ and $\pi_{2}=$ $1-\phi(1-\pi)$. Therefore, for a pessimistic agent, $\pi_{1}<\pi<\pi_{2}$, and for an optimistic agent $\pi_{1}>\pi>\pi_{2}$.

The RDU assumption has two main consequences. The first one corresponds to the deformation of probabilities, that may lead to quantitative changes: with respect to the EU model, the agents behave as if they did not take into account the true probabilities. The second consequence corresponds to the existence of a kink in the indifference curves for $d^{1}=d^{2}$. This property may imply qualitative change in the behaviors of the agents that choose to consume the same level in the two states of the nature for different price levels. In the literature on RDU preferences, it often generates multiple equilibria.

\subsection{The equilibrium without bubbles}

What is the impact of the RDU assumption on the former analysis? Considering the competitive equilibrium without bubbles, it is clear that state 1 is the good state of the nature and state 2 the bad state. Therefore, the utility function is always given by (21). Moreover, the RDU assumption has no impact as the consumption choices do not depend on $\pi$. Consumption and savings remain equal to

$$
\begin{aligned}
c_{t} & =\frac{w}{1+\beta} \\
s_{t} & =\frac{\beta w}{1+\beta} \\
d_{t+1} & =\frac{R\left(\sigma_{t+1}\right) \beta w}{1+\beta}
\end{aligned}
$$


The capital stock from $t=0$ is equal to:

$$
K_{t}=\frac{\beta w}{1+\beta}
$$

What can be said about the Pareto optimality of the equilibrium without bubbles? It is necessary to study separately the cases of pessimistic and optimistic agents. In both cases, the utility function is not differentiable at a point such that $d^{1}=d^{2}$. But, if agents are pessimistic, their preferences remain convex, whereas this property is lost for optimistic agents.

In the first case, it is easy to adapt the result of Proposition 1.

Proposition 4 If agents are pessimistic and

$$
\frac{\pi_{1}}{R_{1}}+\frac{1-\pi_{1}}{R_{2}}<1
$$

the competitive equilibrium without bubbles is interim Pareto optimal.

Proof. See Appendix 2.

This condition is the same as the one of Proposition 1, except that $\pi$ is replaced by $\pi_{1}$. This is due to the transformation of probabilities in the utility function of the agent.

A condition ensuring the Pareto efficiency of the competitive equilibrium without bubbles is more difficult to establish when agents are optimistic, because agents' preferences are no longer convex. The next proposition shows that the preceding efficiency condition remains necessary. But another condition is needed that can be interpreted as a one of "moderate" optimism, or a weak transformation of the probabilities.

Proposition 5 Assuming optimistic agents, if

$$
\frac{\pi_{1}}{R_{1}}+\frac{1-\pi_{1}}{R_{2}}<1
$$

and

$$
\frac{R_{2}}{R_{1}}<\left[\frac{\pi_{1}^{\pi_{2}}\left(1-\pi_{1}\right)^{\left(1-\pi_{2}\right)}}{\pi_{2}^{\pi_{2}}\left(1-\pi_{2}\right)^{\left(1-\pi_{2}\right)}}\right]^{\frac{1}{\pi_{1}-\pi_{2}}}
$$

the competitive equilibrium without bubbles is interim Pareto optimal. 


\section{Proof. See Appendix 3.}

As it is apparent in the proof, Condition (26) is needed because preferences are not convex for an optimistic agent. It guarantees that no other feasible allocation dominating the competitive equilibrium exists in the zone in which $d^{1}<d^{2}$. A better intuition can be achieved in particular cases. The following corollary studies the limit condition obtained from (26) when the transformation of probabilities vanishes. Then, it takes a particular assumption for $\phi$

$$
\phi(\pi)=\pi^{\rho}, \text { with } 0<\rho<1
$$

The lower $\rho$, the more optimistic the agent is.

Corollary 1 1. In the limit case $\pi_{1} \rightarrow \pi$ and $\pi_{2} \rightarrow \pi$, Condition (26) becomes $R_{2} / R_{1}<1$, which is true by assumption. By continuity, (26) is fulfilled if the transformation of probabilities is weak.

2. Let us assume that $\phi(\pi)=\pi^{\rho}$ with $\rho \in(0,1)$. There exists a value $\bar{\rho} \in(0,1)$ such that (26) is satisfied if and only if $\rho>\bar{\rho}$.

Proof. See Appendix 4.

The first part of the corollary shows that Condition (26) is satisfied in the limit case $\pi_{1} \rightarrow \pi$ and $\pi_{2} \rightarrow \pi$. By continuity, it is satisfied if the transformation of probabilities is not too strong. The second part introduces a particular function $\phi$ that allows the transformation of probabilities to be measured by the parameter $\rho$. $\rho$ can be interpreted as the degree of pessimism (or as the opposite of the degree of optimism). The corollary allows a lower bound $\bar{\rho}$ on $\rho$ to be defined that represents a limit value for the degree of optimism.

\subsection{Deterministic bubbles}

For a deterministic bubble, it is clear that second period consumption in state 1 will always be greater than second period consumption in state 2 , as:

$$
d_{t+1}^{1}=R_{1} s_{t}+p_{t+1} x_{t}>d_{t+1}^{2}=R_{2} s_{t}+p_{t+1} x_{t}
$$


Therefore, the analysis of Section 3.1 can be used again, replacing $\pi$ by $\pi_{1}$. A bubbly equilibrium will exist if:

$$
\frac{\pi_{1}}{R_{1}}+\frac{\left(1-\pi_{1}\right)}{R_{2}}>1
$$

It will be associated with a positive investment in capital if

$$
\pi_{1} R_{1}+\left(1-\pi_{1}\right) R_{2}>1
$$

If (28) is not fulfilled, there exists an equilibrium in which the bubble asset is the only asset of the economy, with a price

$$
p=\frac{\beta w}{1+\beta}
$$

Condition (27) defines an upper bound on $\pi_{1}$ :

$$
\pi_{1}<\frac{R_{1}\left(1-R_{2}\right)}{R_{1}-R_{2}}
$$

\section{The case of pessimism}

Agents are assumed to be pessimistic: $\pi_{1}<\pi$. If

$$
\pi_{1}<\frac{R_{1}\left(1-R_{2}\right)}{R_{1}-R_{2}}<\pi
$$

a deterministic bubble exists in an economy in which there would be no bubble if agents did not "transform" the probabilities. The interpretation of this result is simple. Investing in the bubble provides a gross return equal to 1 , which is greater than the capital return in the bad state of the nature $R_{2}$. Agents invest in the bubble in order to be protected against the occurrence of state 2. In the case of pessimism, they put more weight on this state and invest more in the bubble. Therefore, pessimism can play in favor of the existence of a deterministic bubble. From Proposition 4, a bubbly equilibrium can only exist in an inefficient economy.

Figure 2 gives an illustration of the effect of pessimism on the existence of the different regimes in the plane $\left(R_{1}, R_{2}\right)$. The curves are obtained under the assumption that the pessimism generates a transformation of $\pi$ from $\pi=1 / 2$ to $\pi_{1}=0.4$. 


\section{The case of optimism}

The condition ensuring the existence of a bubbly equilibrium remains Condition (27). Optimism is unfavorable to the existence of bubbles, as agents put more weight on the good state of the nature.

The relation between the existence of bubbles and interim Pareto optimality is more complex in the case of optimism. The usual way to analyze the impact of a bubble is to interpret it as an intergenerational transfer. In the basic economy with standard EU preferences, when (5) is not fulfilled, the existence of a bubble constitutes an intergenerational transfer from the young to the old agents and this transfer is Pareto improving. This analysis can also be used to understand the case of pessimistic agents. But, with optimistic agents, preferences are no longer convex. If (25) holds and (26) is not fulfilled, it may be possible that the economy is not interim Pareto optimal and that no bubbly equilibrium exists. Considering the proof of Proposition 5 , the competitive equilibrium without bubbles can be inefficient in this case, because the technology does not allow agents to redistribute consumption from state 1 in favor of state 2. A deterministic bubble cannot solve this problem as the bubble carries out a transfer among generations, and not a transfer among the two states of nature.

\subsection{Stochastic bubbles}

\subsubsection{Two types of bubbly equilibria}

A stochastic bubble conditional to state 2 may induce some redistribution of consumption between the two states of nature. For the equilibrium with a deterministic bubble, state 1 always remains the best state of the nature. For an equilibrium with a stochastic bubble conditional to state 2 , it is possible that state 2 becomes the good state of the nature, as the bubble bursts in state 1 . More precisely, the size of the bubble may determine which is the best state. For a small bubble, state 1 will always lead to more consumption than state 2. For a large bubble, the inequality can be reversed. Therefore, it is a priori possible to obtain two types of bubbly equilibria, associated either with $d^{2}<d^{1}$ or with $d^{2}>d^{1}$.

Assuming that state 2 occurs in period $t$, the budget constraints of a 
generation $t$ agent remain the same:

$$
\begin{aligned}
c_{t}+s_{t}+p_{t} x_{t} & =w \\
d_{t+1}^{1} & =R_{1} s_{t} \\
d_{t+1}^{2} & =R_{2} s_{t}+p_{t+1} x_{t}
\end{aligned}
$$

Equilibrium such that $d^{2}>d^{1}$

Assuming that the equilibrium is such that $d_{t+1}^{2}>d_{t+1}^{1}$, the program of the agent consists in maximizing (22) under the three budget constraints. The results are:

$$
\begin{aligned}
c_{t} & =\frac{w}{1+\beta} \\
s_{t} & =\frac{\beta w}{1+\beta} \frac{\pi_{2}}{1-\frac{p_{t} R_{2}}{p_{t+1}}} \\
x_{t} & =\frac{\beta w}{1+\beta}\left[\frac{\left(1-\pi_{2}\right)}{p_{t}}-\frac{\pi_{2} R_{2}}{p_{t+1}-p_{t} R_{2}}\right]
\end{aligned}
$$

The equilibrium conditions lead to the dynamics of the price of the bubble. Using the variable $\delta_{t}=p_{t}(1+\beta) /(\beta w), \delta_{t}$ follows the dynamic equation:

$$
\delta_{t+1}=R_{2} \delta_{t} \frac{1-\delta_{t}}{1-\pi_{2}-\delta_{t}}
$$

The bubbly steady state corresponds to

$$
\hat{\delta}=\frac{1-\pi_{2}-R_{2}}{1-R_{2}}
$$

It exists only if

$$
\pi_{2}<1-R_{2}
$$

Moreover, the assumption that $d^{1}<d^{2}$ must be checked along this equilibrium. It leads to the constraint:

$$
\pi_{2}<\frac{1-R_{2}}{R_{1}+1-R_{2}}
$$

This last condition is stronger than (29) as $R_{1}+1-R_{2}>1$. 
Equilibrium such that $d^{2}<d^{1}$

Assuming that the equilibrium is such that $d_{t+1}^{2}<d_{t+1}^{1}$, the program of the agent gives:

$$
\begin{aligned}
c_{t} & =\frac{w}{1+\beta} \\
s_{t} & =\frac{\beta w}{1+\beta} \frac{\pi_{1}}{1-\frac{p_{t} R_{2}}{p_{t+1}}} \\
x_{t} & =\frac{\beta w}{1+\beta}\left[\frac{\left(1-\pi_{1}\right)}{p_{t}}-\frac{\pi_{1} R_{2}}{p_{t+1}-p_{t} R_{2}}\right]
\end{aligned}
$$

The variable $\delta_{t}=p_{t}(1+\beta) /(\beta w)$ follows the dynamic equation:

$$
\delta_{t+1}=R_{2} \delta_{t} \frac{1-\delta_{t}}{1-\pi_{1}-\delta_{t}}
$$

The bubbly steady state corresponds to

$$
\check{\delta}=\frac{1-\pi_{1}-R_{2}}{1-R_{2}}
$$

It exists only if

$$
\pi_{1}<1-R_{2}
$$

Moreover, the assumption that $d^{1}>d^{2}$ leads to the constraint that:

$$
\pi_{1}>\frac{1-R_{2}}{R_{1}+1-R_{2}}
$$

\subsubsection{Stochastic bubbles and optimism}

For an optimistic agent, $\pi_{1}>\pi>\pi_{2}$, and thus, $\hat{\delta}>\check{\delta}$. Depending on the value of the parameters, it is possible to obtain a bubbly equilibrium with $d^{1}<d^{2}$ and a high price level of the bubble, or a bubbly equilibrium with $d^{1}>d^{2}$ and a low price level of the bubble. ${ }^{1}$

Using conditions (29), (30), (31) and (32), the following results may be obtained.

Proposition 6 Assume that $\pi_{2}<\frac{1-R_{2}}{R_{1}+1-R_{2}}$ and $\pi_{1}>1-R_{2}$ or $\pi_{1}<\frac{1-R_{2}}{R_{1}+1-R_{2}}$. There exists a unique bubbly equilibrium with $d^{1}<d^{2}$ and a price of the bubble equal to $\hat{\delta}(\beta w) /(1+\beta)$.

\footnotetext{
${ }^{1}$ It is not possible to obtain a bubbly equilibrium such that $d^{1}=d^{2}$, because it is never optimal for the consumer to choose such an allocation in case of optimism.
} 
Proposition 6 shows that optimism can play in favor of the existence of bubbles. Assume that $\pi>1-R_{2}$. Under this condition, stochastic bubbles cannot exist in the economy with EU preferences. If agents are optimistic, it is possible that they transform probabilities with $\pi_{2}=1-\phi(1-\pi)<\frac{1-R_{2}}{R_{1}+1-R_{2}}$. In this case a stochastic bubble may exist. The price of the bubble is high enough in such a way that consumption in state 2 (the bubble exists) is higher than consumption in state 1 (the bubble explodes). As agents are optimistic, they put more weight on the good state and invest more in the bubble.

Proposition 7 corresponds to the converse case of a low price of the bubble such that $d^{1}$ remains higher than $d^{2}$.

Proposition 7 Assume that $\pi_{1}<1-R_{2}$ and $\pi_{2}>\frac{1-R_{2}}{R_{1}+1-R_{2}}$. There exists a unique bubbly equilibrium with $d^{1}>d^{2}$ and a price of the bubble equal to $\check{\delta}(\beta w) /(1+\beta)$.

As $\pi<\pi_{1}<1-R_{2}$, a necessary condition for the existence of such an equilibrium is that there exists a bubbly equilibrium in the economy with EU preferences (see condition (20)). Moreover agents need to be not too optimistic, in such a way that $\pi_{1}$ and $\pi_{2}$ remain in the interval $\left(\frac{1-R_{2}}{R_{1}+1-R_{2}}, 1-R_{2}\right)$.

A last case remains to be studied, if

$$
\pi_{2}<\frac{1-R_{2}}{R_{1}+1-R_{2}}<\pi_{1}<1-R_{2}
$$

In this case, all preceding conditions (29), (30), (31) and (32) are fulfilled. But, it does not imply that the two types of bubbly equilibria can exist together. Indeed, in the case of optimistic agents, preferences are not convex. It is possible that two different solutions satisfy the marginal conditions of the consumer program, one with $d^{1}<d^{2}$, the other one with $d^{1}>d^{2}$. Therefore, it is necessary to compare the utility levels associated with the two solutions. This is done in the following proposition.

Proposition 8 The function $\zeta$ is defined according to:

$$
\zeta(\pi)=\pi \ln \left(\pi \frac{R_{1}}{1-R_{2}}\right)+(1-\pi) \ln (1-\pi)
$$


Assuming

$$
\pi_{2}<\frac{1-R_{2}}{R_{1}+1-R_{2}}<\pi_{1}<1-R_{2}
$$

there exists two cases:

- If $\zeta\left(\pi_{2}\right)>\zeta\left(\pi_{1}\right)$ then a bubbly equilibrium with $d^{1}<d^{2}$ exists and the price of the bubble is equal to $\hat{\delta}(\beta w) /(1+\beta)$.

- If $\zeta\left(\pi_{2}\right)<\zeta\left(\pi_{1}\right)$ then a bubbly equilibrium with $d^{1}>d^{2}$ exists and the price of the bubble is equal to $\check{\delta}(\beta w) /(1+\beta)$.

Proof. See Appendix 5.

To summarize the impact of optimism on the existence of stochastic bubbles, the most interesting result is obtained in Proposition 6. Optimism may favor stochastic bubbles with respect to EU preferences. The price of the bubble must be high enough in such a way that $d^{1}<d^{2}$. In that case, state 2 (the bubble exists) can be better than state 1 (the bubble bursts). Optimistic agents put more weight on the good state and invest more in the bubble.

\subsubsection{Stochastic bubbles and pessimism}

For a pessimistic agent, $\pi_{1}<\pi<\pi_{2}$. The preceding conditions (29), (30), (31) and (32) allow existence conditions for the bubbly equilibria to be obtained that satisfy the marginal conditions of the consumer program. But, in the case of pessimism, another situation may exist that corresponds to $d_{t+1}^{1}=d_{t+1}^{2}$. This case results from the existence of a kink for $d_{t+1}^{1}=d_{t+1}^{2}$.

Considering the maximization of the RDU function under the intertemporal budget constraint (39), the optimal solution with $d_{t+1}^{1}=d_{t+1}^{2}$ is obtained if:

$$
\frac{\pi_{1}}{1-\pi_{1}}<\frac{1-R_{2} \frac{p_{t}}{p_{t+1}}}{R_{1} \frac{p_{t}}{p_{t+1}}}<\frac{\pi_{2}}{1-\pi_{2}}
$$

It corresponds to the solution:

$$
\begin{aligned}
c_{t} & =\frac{w}{1+\beta} \\
d_{t+1}^{1} & =d_{t+1}^{2}=\frac{\frac{\beta w}{1+\beta}}{\frac{1}{R_{1}}-\frac{R_{2}}{R_{1}} \frac{p_{t}}{p_{t+1}}+\frac{p_{t}}{p_{t+1}}}
\end{aligned}
$$


The equilibrium price of the bubble satisfies:

$$
p_{t}=\frac{\beta w}{1+\beta} \frac{\frac{p_{t}}{p_{t+1}}\left(R_{1}-R_{2}\right)}{1+\frac{p_{t}}{p_{t+1}}\left(R_{1}-R_{2}\right)}
$$

With the change of variable $\delta_{t}=p_{t}(1+\beta) /(\beta w)$, this equation gives:

$$
\begin{aligned}
\delta_{t} & =0 \text { or } \\
\delta_{t+1} & =-\delta_{t}\left(R_{1}-R_{2}\right)+\left(R_{1}-R_{2}\right)
\end{aligned}
$$

(33) has a stationary state

$$
\tilde{\delta}=\frac{R_{1}-R_{2}}{1+R_{1}-R_{2}}
$$

If $R_{1}-R_{2}>1$, this stationary state is unstable. If $R_{1}-R_{2}<1$, it is stable. In this case, it is possible to observe convergence towards the stationary state, with oscillations. In the case of instability, there is only one stationary bubbly equilibrium. In the case of stability, a multiplicity of bubbly equilibria exists.

Finally, the following results have been obtained in the case of a pessimistic agent:

Proposition 9 Assume that $\pi_{1}<\pi<\pi_{2}$.

- If

$$
\pi_{2}<\frac{1-R_{2}}{R_{1}+1-R_{2}}
$$

a bubbly equilibrium with $d^{1}<d^{2}$ exists and the price of the bubble is equal to $\hat{\delta}(\beta w) /(1+\beta)$.

- If

$$
\frac{1-R_{2}}{R_{1}+1-R_{2}}<\pi_{1}<1-R_{2}
$$

a bubbly equilibrium with $d^{1}>d^{2}$ exists and the price of the bubble is equal to $\check{\delta}(\beta w) /(1+\beta)$.

- If

$$
\pi_{1}<\frac{1-R_{2}}{R_{1}+1-R_{2}}<\pi_{2}
$$

a stationary bubbly equilibrium exists associated with a price of the bubble $\tilde{\delta}(\beta w) /(1+\beta)$. 
- If $R_{1}-R_{2}>1$, this stationary state is unstable.

- If $R_{1}-R_{2}<1$, it is stable. In this case, a multiplicity of bubbly equilibria exists that converge towards the steady state with oscillations.

To summarize, pessimism may favor stochastic bubbles such that $d^{1}>$ $d^{2}$ with respect to EU preferences. To illustrate this point, assume that $\pi>1-R_{2}$. Under this condition, stochastic bubbles cannot exist in the economy with EU preferences. If agents are pessimistic, it is possible that they transform probabilities in such a way that $\pi_{1}=\phi(\pi)<1-R_{2}$. In this case a stochastic bubble can exist. The price of the bubble is low enough in such a way that consumption in state 2 (the bubble exists) remains lower than consumption in state 1 (the bubble explodes). As agents are pessimistic, they put more weight on the bad state and invest more in the bubble.

Moreover it is possible to have indeterminacy with a multiplicity of bubbly equilibria converging towards a steady state with oscillations. This result of indeterminacy is related to the existence of a kink on the indifference curves for $d^{1}=d^{2}$. When $d^{1}=d^{2}$, it is possible that different values of the price of the bubble are compatible with an equilibrium. Similar results have been obtained with RDU preferences or Choquet utility in other frameworks: Tallon (1997) and Epstein and Wang (1994) also demonstrate the existence of multiple equilibria in models of financial assets.

\section{Conclusion}

This paper has proposed a simple model that suggests that uncertainty associated with RDU preferences can extend the scope for the existence of rational financial bubbles. Pessimism favors the existence of deterministic bubbles, when optimism may promote the existence of stochastic bubbles. Moreover, associated with pessimism, the RDU assumption is a new cause of multiple bubbly equilibria.

It would be interesting to expand these first results into a more general framework. A first improvement would consist in introducing different 
production technologies subject to different shocks, with many states of the nature. Considering non-linear production technologies could also be an interesting generalization.

Another development would be to assume heterogeneous agents differing by their degree of pessimism or optimism.

\section{References}

[1] Abel, Andrew B, N. Gregory Mankiw, Lawrence H. Summers and Richard J. Zeckhauser, 1989. "Assessing Dynamic Efficiency: Theory and Evidence," Review of Economic Studies, Wiley Blackwell, vol. 56(1), pages 1-19, January.

[2] Allais, Maurice, Economie et intérêt, ed. Imprimerie Nationale, 1947

[3] Bosi, Stefano and Thomas Seegmuller, On rational exuberance, Mathematical Social Sciences, Volume 59, Issue 2, March 2010, Pages 249-270

[4] Ricardo J. Caballero \& Mohamad L. Hammour, 2002. "Speculative Growth," NBER Working Papers 9381, National Bureau of Economic Research, Inc.

[5] Caballero, Ricardo J., Emmanuel Farhi and Mohamad Hammour, 2006, Speculative Growth: Hints from the U.S. Economy, American Economic Review, vol 96(4): 1159-1192.

[6] Chateauneuf, A. "Comonotonicity axioms and RDU theory for arbitrary consequences", Journal of Mathematical Economics, 32, 21-45, 1999.

[7] Cohen, M. and J M Tallon, "Décision dans le risque et l'incertain: l'apport des modèles non-additifs", Revue d'Economie Politique, $\mathrm{N}^{\circ} 110(5), 2000$, pp.631-681.

[8] De La Croix, David and Philippe Michel, A theory of economic growth: dynamics and policy in overlapping generations, Cambridge University Press; (2002). 
[9] Demange, Gabrielle and Guy Laroque, Social Security and Demographic Shocks, Econometrica, Vol. 67, No. 3 (May, 1999), pp. 527-542

[10] Diamond, Peter, 1965, National Debt in a Neoclassical Growth Model, American Economic Review, vol 55(5): 1126-50.

[11] Dow, James \& da Costa Werlang, Sergio Ribeiro, 1992. "Excess volatility of stock prices and Knightian uncertainty," European Economic Review, Elsevier, vol. 36(2-3), pages 631-638, April.

[12] Larry G. Epstein and Tan Wang, Intertemporal Asset Pricing under Knightian Uncertainty, Econometrica, Vol. 62, No. 2 (Mar., 1994), pp. 283-322

[13] Piero Gottardi, Stationary Monetary Equilibria in Overlapping Generations Models with Incomplete Markets, Journal of Economic Theory 71, 7589 (1996).

[14] Grossman, Gene and Noriyuki Yanagawa, 1993, Asset Bubbles and Endogenous Growth, Journal of Monetary Economics, vol 31(1): 3-19.

[15] Homburg, Stefan. Efficient Economic Growth. Berlin usw. Springer, 1992.

[16] Olivier, Jacques, 2000, Growth-enhancing Bubbles, International Economic Review, vol 41(1): 133-151.

[17] Peled, Dan, Stationary Pareto Optimality of Stochastic Asset Equilibria with Overlapping Generations, Journal of Economic Theory 34, 396-403 (1984).

[18] Peled, Dan, and Rao Aiyagari, Dominant Root Characterization of Pareto Optimality and the Existence of Optimal Equilibria in Stochastic Overlapping Generations Models, Journal of Economic Theory 54, 69-83 (1991).

[19] Quiggin, J. "A theory of anticipated utility",Journal of Economic Behavior and Organisation, 3, p. 323-343, 1982. 
[20] Samuelson, Paul, 1958, An Exact Consumption-Loan Model of Interest with or without the Social Contrivance of Money, Journal of Political Economy, vol 66: 467-482.

[21] Tallon, J M, Risque microéconomique et prix d'actifs dans un modèle d'équilibre général avec espérance d'utilité dépendante du rang Finance, Revue de l'Association Française de Finance, N 18, 1997, pp. 139-153.

[22] Tirole, Jean (1985): "Asset Bubbles and Overlapping Generations," Econometrica, 53(6), 1499-1528.

[23] Wang, Y. (1993): "Stationary Equilibria in an Overlapping Generations Economy with Stochastic Production," Journal of Economic Theory, 61, 423-435.

[24] Weil, Philippe, 1987, Confidence in the Real Value of Money in an Overlapping Generations Economy, Quarterly Journal of Economics, vol 52(1): 1-22.

\section{Appendixes}

\subsection{Appendix 1}

Proof of Proposition 1.

The proof uses the method developed by Homburg (1992) and De la Croix and Michel (2002). For the proof, it is useful to introduce some notations. $h_{t}$ denotes a particular history from period 0 till $t$ (the state $\sigma_{0}$ at period 0 is assumed to be known in $t=0): h_{t}=\left(\sigma_{0}, \sigma_{1}, \sigma_{2}, \ldots, \sigma_{t}\right) . H_{t}$ is the set of all possible $t$-period histories from period 0 . \# $H_{t}=2^{t}$ as $\sigma_{0}$ is known in 0 . The applications $\tau$ and $\theta$ are defined such that, for an history $h_{t}=$ $\left(\sigma_{0}, \sigma_{1}, \sigma_{2}, \ldots, \sigma_{t-1}, \sigma_{t}\right) \in H_{t}, \tau\left(h_{t}\right)=\left(\sigma_{0}, \sigma_{1}, \sigma_{2}, \ldots, \sigma_{t-1}\right)$ and $\theta\left(h_{t}\right)=\sigma_{t}$.

The allocation corresponding to the competitive equilibrium is such that, 
for all $t \geq 0$ :

$$
\begin{aligned}
& K_{-1} \text { is given } \\
d_{0}= & R\left(\sigma_{0}\right) K_{-1} \\
c_{t}= & \frac{w}{1+\beta} \equiv \bar{c} \\
K_{t}= & \frac{\beta w}{1+\beta} \equiv \bar{K} \\
d_{t+1}= & \frac{R\left(\sigma_{t+1}\right) \beta w}{1+\beta}
\end{aligned}
$$

Using the following notation,

$$
\bar{d}\left(\sigma_{t}\right)=\frac{R\left(\sigma_{t}\right) \beta w}{1+\beta}
$$

the corresponding ex-ante utility level is:

$$
\bar{U} \equiv \ln (\bar{c})+\beta \pi \ln (\bar{d}(1))+\beta(1-\pi) \ln (\bar{d}(2))
$$

Assuming that this allocation is not interim Pareto optimal means that there exists another feasible allocation that, almost surely, gives a higher expected utility for all period $t$ with a strict improvement on a set of states of positive measure. Formally, it means that it is possible to find an allocation $\left(\tilde{c}\left(h_{t}\right), \tilde{d}\left(h_{t}\right), \tilde{K}\left(h_{t}\right)\right)_{h_{t} \in H_{t}, t \geq 0}$ such that $\forall t$

$$
\begin{aligned}
\tilde{c}\left(h_{t}\right)+\tilde{d}\left(h_{t}\right)+\tilde{K}\left(h_{t}\right) & =R\left(\theta\left(h_{t}\right)\right) \tilde{K}\left(\tau\left(h_{t}\right)\right)+w \\
\tilde{K}\left(\tau\left(h_{0}\right)\right) & =K_{-1} \text { (initial condition given) }
\end{aligned}
$$

(feasibility), and such that $\forall t, \forall h_{t} \in H_{t}$

$$
\begin{aligned}
\ln \left(\tilde{c}\left(h_{t}\right)\right)+\beta \pi \ln \left(\tilde{d}\left(\left(h_{t}, 1\right)\right)\right)+\beta(1-\pi) \ln \left(\tilde{d}\left(\left(h_{t}, 2\right)\right)\right) & \geq \bar{U} \\
\tilde{d}\left(\sigma_{0}\right) & \geq R\left(\sigma_{0}\right) K_{d}(35)
\end{aligned}
$$

with a strict inequality for some $h_{t_{0}}$.

First, it is easy to check that the competitive solution $(\bar{c}, \bar{d}(1), \bar{d}(2))$ in period $t$ can be obtained through the following program:

$$
\begin{aligned}
\max _{\left(c, d^{1}, d^{2}\right)} \ln (c)+\beta \pi \ln \left(d^{1}\right)+\beta(1-\pi) \ln \left(d^{2}\right) \\
\text { s.t. } w=c+\frac{\pi}{R_{1}} d^{1}+\frac{(1-\pi)}{R_{2}} d^{2}
\end{aligned}
$$


As a consequence of this property, (34) implies that, $\forall t, \forall h_{t} \in H_{t}$

$$
\tilde{c}\left(h_{t}\right)+\frac{\pi}{R_{1}} \tilde{d}\left(\left(h_{t}, 1\right)\right)+\frac{(1-\pi)}{R_{2}} \tilde{d}\left(\left(h_{t}, 2\right)\right) \geq \bar{c}+\frac{\pi}{R_{1}} \bar{d}(1)+\frac{(1-\pi)}{R_{2}} \bar{d}(2)
$$

with a strict inequality for some history $h_{t_{0}}$.

For a state $\sigma_{t}, \sigma_{t} \in\{1,2\}$, the function $\chi$ is defined as:

$$
\begin{aligned}
& \chi(1)=\pi \\
& \chi(2)=1-\pi
\end{aligned}
$$

For an history $h_{t}=\left(\sigma_{0}, \sigma_{1}, \sigma_{2}, \ldots, \sigma_{t-1}, \sigma_{t}\right) \in H_{t}, P\left(h_{t}\right)$ is defined as

$$
P\left(h_{t}\right)=\frac{\prod_{i=1}^{t} \chi\left(\sigma_{i}\right)}{\prod_{i=1}^{t} R\left(\sigma_{i}\right)}
$$

and $P\left(\sigma_{0}\right)=1$. Therefore,

$$
P\left(h_{t}\right)=\frac{\chi\left(\theta\left(h_{t}\right)\right)}{R\left(\theta\left(h_{t}\right)\right)} P\left(\tau\left(h_{t}\right)\right)
$$

For $T>t_{0}$, it is obtained that

$$
\begin{aligned}
& \tilde{d}\left(\sigma_{0}\right)-d_{0}+ \\
& \sum_{t=0}^{T-1} \sum_{h_{t} \in H_{t}} P\left(h_{t}\right)\left[\tilde{c}\left(h_{t}\right)+\frac{\pi}{R_{1}} \tilde{d}\left(\left(h_{t}, 1\right)\right)+\frac{(1-\pi)}{R_{2}} \tilde{d}\left(\left(h_{t}, 2\right)\right)-\bar{c}-\frac{\pi}{R_{1}} \bar{d}(1)-\frac{(1-\pi)}{R_{2}} \bar{d}(2)\right]>0
\end{aligned}
$$

Rearranging the terms that depend on the same period, the following is obtained:

$$
\begin{aligned}
& \sum_{t=0}^{T-1} \sum_{h_{t} \in H_{t}} P\left(h_{t}\right)\left[\tilde{c}\left(h_{t}\right)+\tilde{d}\left(h_{t}\right)-\bar{c}-\bar{d}\left(\theta\left(h_{t}\right)\right)\right]+ \\
& \sum_{h_{T} \in H_{T}} P\left(h_{T}\right)\left[\tilde{d}\left(\left(h_{T}\right)\right)-\bar{d}\left(\theta\left(h_{T}\right)\right)\right]>0
\end{aligned}
$$

From the feasibility constraints, it is obtained:

$$
\begin{aligned}
& \sum_{t=0}^{T-1} \sum_{h_{t} \in H_{t}} P\left(h_{t}\right)\left[R\left(\theta\left(h_{t}\right)\right) \tilde{K}\left(\tau\left(h_{t}\right)\right)-\tilde{K}\left(h_{t}\right)-R\left(\theta\left(h_{t}\right)\right) \bar{K}+\bar{K}\right]+ \\
& \sum_{h_{T} \in H_{T}} P\left(h_{T}\right)\left[\tilde{d}\left(\left(h_{T}\right)\right)-\bar{d}\left(\theta\left(h_{T}\right)\right)\right]>0
\end{aligned}
$$


After simplifications, it is obtained:

$$
\sum_{h_{T-1} \in H_{T-1}} P\left(h_{T-1}\right)\left[-\tilde{K}\left(h_{T-1}\right)+\bar{K}\right]+\sum_{h_{T} \in H_{T}} P\left(h_{T}\right)\left[\tilde{d}\left(\left(h_{T}\right)\right)-\bar{d}\left(\theta\left(h_{T}\right)\right)\right]>0
$$

It is possible to write:

$$
-\tilde{K}\left(h_{T-1}\right)+\bar{K}=\frac{\pi}{R(1)} R(1)\left[-\tilde{K}\left(h_{T-1}\right)+\bar{K}\right]+\frac{1-\pi}{R(2)} R(2)\left[-\tilde{K}\left(h_{T-1}\right)+\bar{K}\right]
$$

Replacing in (36) makes it possible to write:

$$
\sum_{h_{T} \in H_{T}} P\left(h_{T}\right)\left[\tilde{d}\left(h_{T}\right)-R\left(\theta\left(h_{T}\right)\right) \tilde{K}\left(\tau\left(h_{T}\right)\right)-\bar{d}\left(\theta\left(h_{T}\right)\right)+R\left(\theta\left(h_{T}\right)\right) \bar{K}\right]>0
$$

Using the feasibility constraint leads to:

$$
\sum_{h_{T} \in H_{T}} P\left(h_{T}\right)\left[\bar{c}+\bar{K}-\tilde{c}\left(h_{T}\right)-\tilde{K}\left(h_{T}\right)\right]>0
$$

Finally, $\bar{c}+\bar{K}=w$ and it is obtained:

$$
\left[\sum_{h_{T} \in H_{T}} P\left(h_{T}\right)\right] w>\sum_{h_{T} \in H_{T}} P\left(h_{T}\right)\left[\bar{c}+\bar{K}-\tilde{c}\left(h_{T}\right)-\tilde{K}\left(h_{T}\right)\right]>0
$$

Introducing the notation

$$
S_{T}=\sum_{h_{T} \in H_{T}} P\left(h_{T}\right)
$$

it is straightforward to check that

$$
S_{T}=\left(\frac{\pi}{R_{1}}+\frac{1-\pi}{R_{2}}\right) S_{T-1}
$$

Therefore, if $\frac{\pi}{R_{1}}+\frac{1-\pi}{R_{2}}<1, \lim _{T \rightarrow \infty} S_{T}=0$ and the competitive equilibrium is interim Pareto-optimal.

\subsection{Appendix 2}

The proof is adapted from the preceding one, replacing $\pi$ by $\pi_{1}$. The competitive solution in period $t$ can be obtained through the following program:

$$
\begin{aligned}
\max _{\left(c, d^{1}, d^{2}\right)} \ln (c)+\beta \pi_{1} \ln \left(d^{1}\right)+\beta\left(1-\pi_{1}\right) \ln \left(d^{2}\right) \\
\text { s.t. } w=c+\frac{\pi_{1}}{R_{1}} d^{1}+\frac{\left(1-\pi_{1}\right)}{R_{2}} d^{2}
\end{aligned}
$$


As the agent is pessimistic, its preferences remain strictly convex. Therefore, the preceding reasoning can be used: a feasible allocation $\left(\tilde{c}\left(h_{t}\right), \tilde{d}\left(h_{t}\right)\right.$, $\left.\tilde{K}\left(h_{t}\right)\right)_{h_{t} \in H_{t}, t \geq 0}$ that interim Pareto-dominates the competitive equilibrium must satisfy $\forall t, \forall h_{t} \in H_{t}$

$$
\tilde{c}\left(h_{t}\right)+\frac{\pi_{1}}{R_{1}} \tilde{d}\left(\left(h_{t}, 1\right)\right)+\frac{\left(1-\pi_{1}\right)}{R_{2}} \tilde{d}\left(\left(h_{t}, 2\right)\right) \geq \bar{c}+\frac{\pi_{1}}{R_{1}} \bar{d}(1)+\frac{\left(1-\pi_{1}\right)}{R_{2}} \bar{d}(2)
$$

with a strict inequality for some history $h_{t_{0}}$. Thereafter, the proof is the same, replacing $\pi$ by $\pi_{1}$.

\subsection{Appendix 3}

The proof is adapted from Appendix 1. The competitive solution in period $t$ can be obtained through the following program:

$$
\begin{aligned}
\max _{\left(c, d^{1}, d^{2}\right)} \ln (c)+\beta \pi_{1} \ln \left(d^{1}\right)+\beta\left(1-\pi_{1}\right) \ln \left(d^{2}\right) \\
\text { s.t. } w=c+\frac{\pi_{1}}{R_{1}} d^{1}+\frac{\left(1-\pi_{1}\right)}{R_{2}} d^{2}
\end{aligned}
$$

But, as the agent is optimistic, its preferences are no more convex. More precisely, it is possible that the program

$$
\begin{array}{r}
\max _{\left(c, d^{1}, d^{2}\right)} \ln c+\beta E_{\phi} \ln (d) \\
\text { s.t. } w=c+\frac{\pi_{1}}{R_{1}} d^{1}+\frac{\left(1-\pi_{1}\right)}{R_{2}} d^{2}
\end{array}
$$

has its optimal solution in the domain $d^{1}<d^{2}$. In this case the solution results from the program

$$
\begin{aligned}
\max _{\left(c, d^{1}, d^{2}\right)} \ln (c)+\beta \pi_{2} \ln \left(d^{1}\right)+\beta\left(1-\pi_{2}\right) \ln \left(d^{2}\right) \\
\text { s.t. } w=c+\frac{\pi_{1}}{R_{1}} d^{1}+\frac{\left(1-\pi_{1}\right)}{R_{2}} d^{2}
\end{aligned}
$$


The solution is:

$$
\begin{aligned}
c & =\frac{w}{1+\beta} \\
d^{1} & =\frac{\pi_{2}}{\pi_{1}} \frac{R_{1} \beta w}{1+\beta} \\
d^{2} & =\frac{1-\pi_{2}}{1-\pi_{1}} \frac{R_{2} \beta w}{1+\beta}
\end{aligned}
$$

It exists only if $d^{1}<d^{2}$, which implies:

$$
\frac{\pi_{2}}{\pi_{1}} R_{1}<\frac{1-\pi_{2}}{1-\pi_{1}} R_{2}
$$

Moreover, it is the optimal solution only if the level of utility is higher than the level for the competitive equilibrium. This condition gives the inequality:

$$
\begin{aligned}
& \ln \left(\frac{w}{1+\beta}\right)+\beta \pi_{2} \ln \left(\frac{\pi_{2}}{\pi_{1}} \frac{R_{1} \beta w}{1+\beta}\right)+\beta\left(1-\pi_{2}\right) \ln \left(\frac{1-\pi_{2}}{1-\pi_{1}} \frac{R_{2} \beta w}{1+\beta}\right) \\
> & \ln \left(\frac{w}{1+\beta}\right)+\beta \pi_{1} \ln \left(\frac{R_{1} \beta w}{1+\beta}\right)+\beta\left(1-\pi_{1}\right) \ln \left(\frac{R_{2} \beta w}{1+\beta}\right)
\end{aligned}
$$

that can be simplified through:

$$
\frac{R_{2}}{R_{1}}>\left[\frac{\pi_{1}^{\pi_{2}}\left(1-\pi_{1}\right)^{\left(1-\pi_{2}\right)}}{\pi_{2}^{\pi_{2}}\left(1-\pi_{2}\right)^{\left(1-\pi_{2}\right)}}\right]^{\frac{1}{\pi_{1}-\pi_{2}}}
$$

It is easy to check that this last condition is stronger than (38). Therefore, if the converse condition (26) is satisfied, the competitive equilibrium is the solution of the program (37). The reasoning of Appendix 1 can now be used: under (26), a feasible allocation $\left(\tilde{c}\left(h_{t}\right), \tilde{d}\left(h_{t}\right), \tilde{K}\left(h_{t}\right)\right)_{h_{t} \in H_{t}, t \geq 0}$ that interim Pareto-dominates the competitive equilibrium must satisfy $\forall t, \forall h_{t} \in H_{t}$

$$
\tilde{c}\left(h_{t}\right)+\frac{\pi_{1}}{R_{1}} \tilde{d}\left(\left(h_{t}, 1\right)\right)+\frac{\left(1-\pi_{1}\right)}{R_{2}} \tilde{d}\left(\left(h_{t}, 2\right)\right) \geq \bar{c}+\frac{\pi_{1}}{R_{1}} \bar{d}(1)+\frac{\left(1-\pi_{1}\right)}{R_{2}} \bar{d}(2)
$$

with a strict inequality for some history $h_{t_{0}}$. Thereafter, the proof is the same, replacing $\pi$ by $\pi_{1}$. 


\subsection{Appendix 4}

To prove the first part of corollary (1), a limited development of the right hand side of (26) is made, taking the ln:

$$
\begin{gathered}
\ln \left[\frac{\pi_{1}^{\pi_{2}}\left(1-\pi_{1}\right)^{\left(1-\pi_{2}\right)}}{\pi_{2}^{\pi_{2}}\left(1-\pi_{2}\right)^{\left(1-\pi_{2}\right)}}\right]^{\frac{1}{\pi_{1}-\pi_{2}}}=\frac{1}{\pi_{1}-\pi_{2}}\left[\pi_{2} \ln \frac{\pi_{1}}{\pi_{2}}+\left(1-\pi_{2}\right) \ln \left(\frac{1-\pi_{1}}{1-\pi_{2}}\right)\right] \\
\sim \frac{1}{\pi_{1}-\pi_{2}}\left\{\pi_{2}\left[\frac{\pi_{1}-\pi_{2}}{\pi_{2}}-\frac{1}{2}\left(\frac{\pi_{1}-\pi_{2}}{\pi_{2}}\right)^{2}\right]+\left(1-\pi_{2}\right)\left[-\left(\frac{\pi_{1}-\pi_{2}}{1-\pi_{2}}\right)-\frac{1}{2}\left(\frac{\pi_{1}-\pi_{2}}{1-\pi_{2}}\right)^{2}\right]\right\} \\
=\frac{1}{\pi_{1}-\pi_{2}}\left[-\frac{1}{2}\left(\pi_{1}-\pi_{2}\right)^{2}\left(\frac{1}{\pi_{2}}+\frac{1}{1-\pi_{2}}\right)\right] \\
=-\frac{1}{2}\left(\pi_{1}-\pi_{2}\right)\left(\frac{1}{\pi_{2}}+\frac{1}{1-\pi_{2}}\right)
\end{gathered}
$$

This last expression tends to 0 when $\pi_{1}-\pi_{2} \rightarrow 0$. Therefore, condition (26) tends to

$$
R_{2} / R_{1}<1
$$

For proving the second part of the corollary, condition (26) can be written:

$$
\frac{R_{2}}{R_{1}}<\left[\frac{\left(\pi^{\rho}\right)^{1-(1-\pi)^{\rho}}\left(1-\pi^{\rho}\right)^{(1-\pi)^{\rho}}}{\left(1-(1-\pi)^{\rho}\right)^{1-(1-\pi)^{\rho}}\left((1-\pi)^{\rho}\right)^{(1-\pi)^{\rho}}}\right]^{\frac{1}{\pi^{\rho}-1+(1-\pi)^{\rho}}}
$$

For a given value of $\pi$, the right hand side of the inequality is an increasing function of $\rho$ that maps $(0,1)$ to $(0,1)$. Therefore, $(26)$ allows a lower bound on $\rho$ to be defined.

\subsection{Appendix 5: proof of Proposition 8}

The intertemporal budget constraint of the agent is obtained with (15), (16), and (17):

$$
c_{t}+\frac{d_{t+1}^{1}}{R_{1}}\left(1-R_{2} \frac{p_{t}}{p_{t+1}}\right)+d_{t+1}^{2} \frac{p_{t}}{p_{t+1}}=w
$$

For a steady state, the constraint becomes:

$$
c+\frac{d^{1}}{R_{1}}\left(1-R_{2}\right)+d^{2}=w
$$


that does not depend on the value of the bubble asset $p$ and only depends on exogenous variables. To determine if the optimal choice of the consumer is obtained with $d^{1}>d^{2}$ or $d^{1}<d^{2}$, it is necessary to compare the indirect utilities associated with the two cases. It is straightforward to calculate that the optimal solution is associated with $d^{1}<d^{2}$ iff:

$\pi_{2} \ln \left(\pi_{2} \frac{R_{1}}{1-R_{2}}\right)+\left(1-\pi_{2}\right) \ln \left(1-\pi_{2}\right)>\pi_{1} \ln \left(\pi_{1} \frac{R_{1}}{1-R_{2}}\right)+\left(1-\pi_{1}\right) \ln \left(1-\pi_{1}\right)$

The function

$$
\zeta(\pi)=\pi \ln \left(\pi \frac{R_{1}}{1-R_{2}}\right)+(1-\pi) \ln (1-\pi)
$$

reaches it minimum value for

$$
\pi=\frac{1-R_{2}}{R_{1}+1-R_{2}}
$$

Finally, the results of Proposition 8 are obtained. 


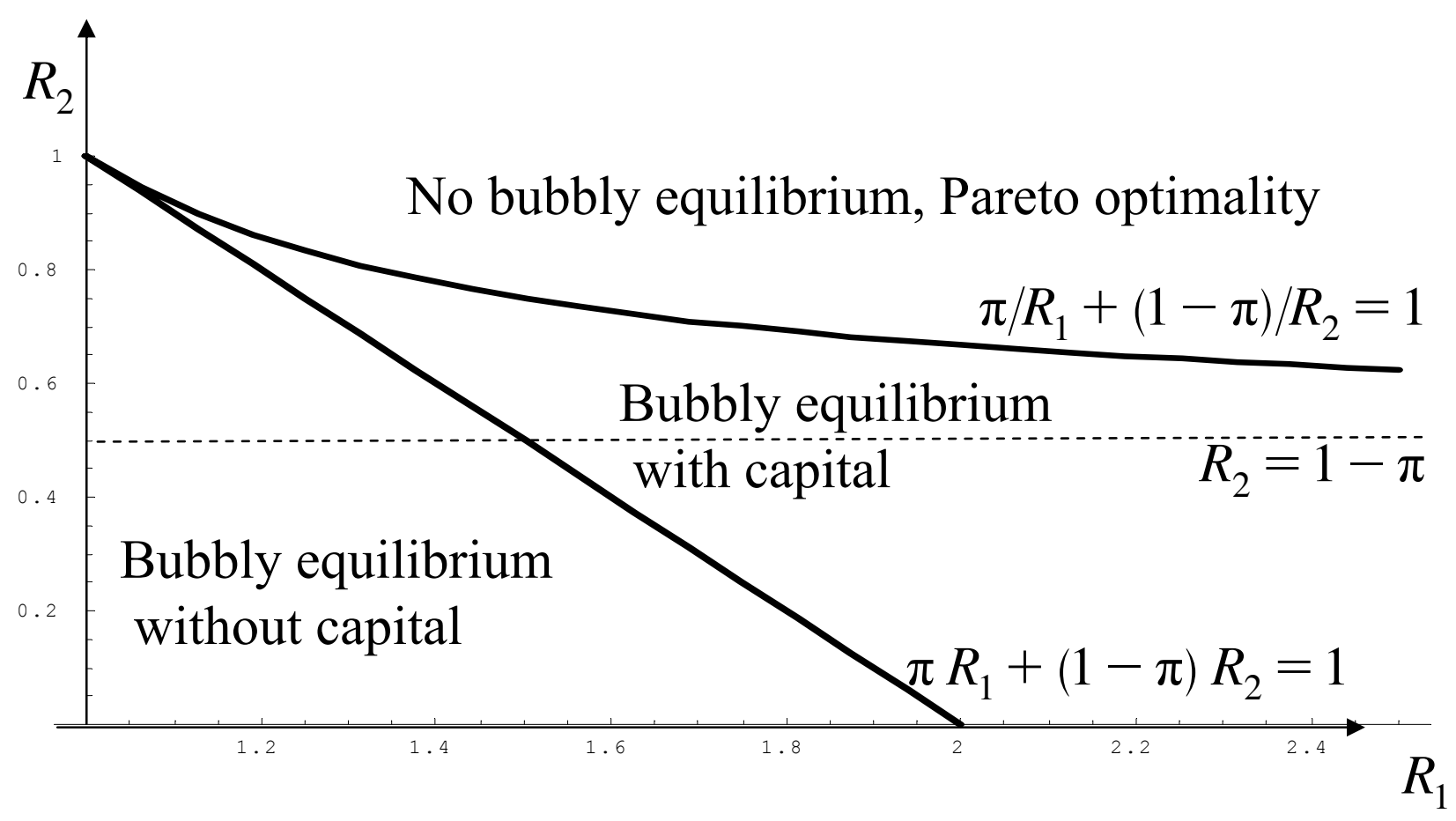

Fig 1: existence of bubbly equilibria with respect to $R_{1}$ and $R_{2}$ 


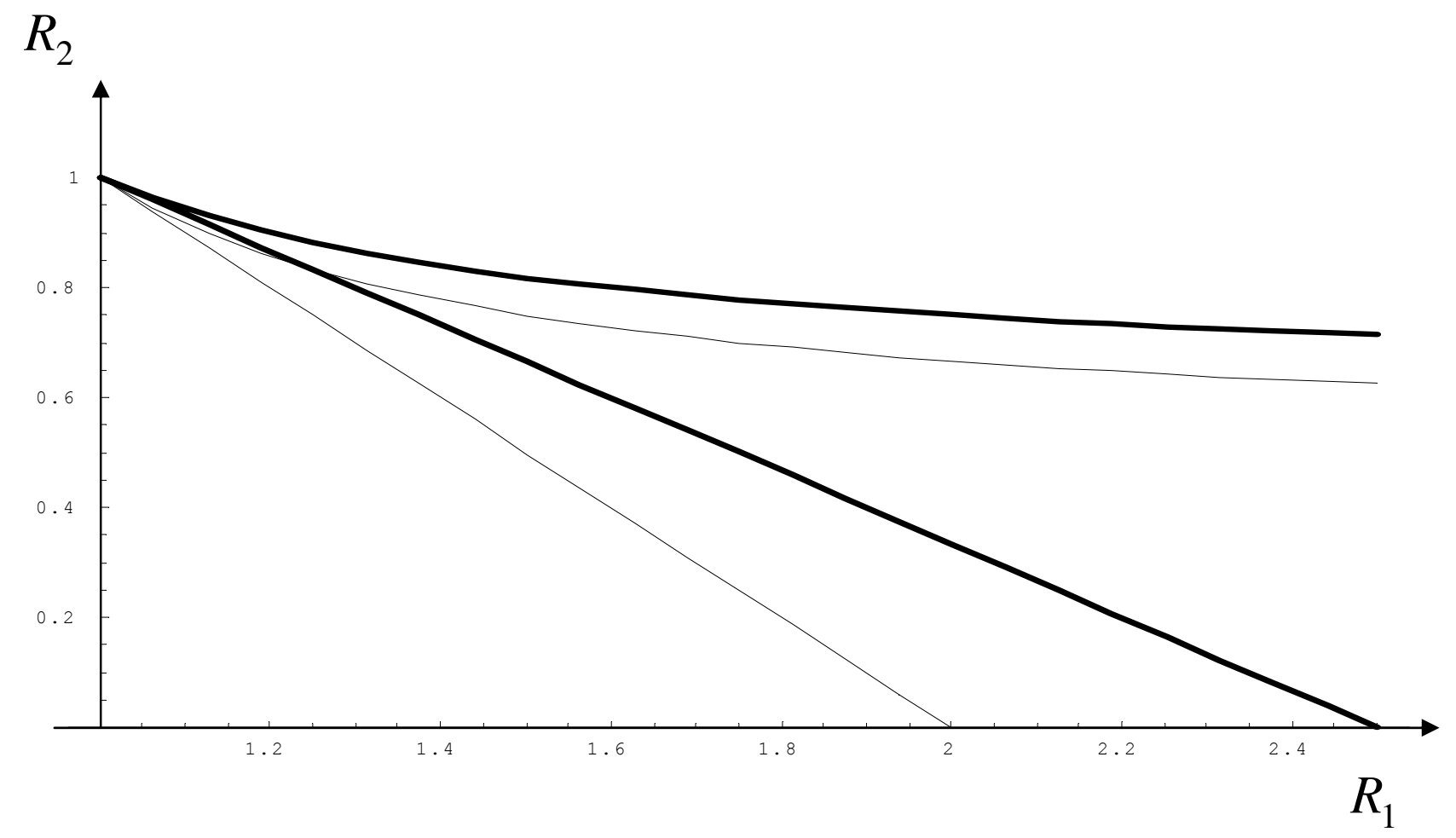

Fig 2: effect of pessimism on the existence of bubbly equilibria 